\title{
Efficacy and Safety of Low-Dose Intravenous Versus Intramuscular Vitamin $K$ in Parenteral Nutrition Patients
}

\author{
Gregory P. Schepers, Pharm.D., Anne R. Dimitry, R.Ph., Frederic E. Eckhauser, M.D., ANd \\ Duane M. Kirking, Pharm.D., Ph.D.

\begin{abstract}
From the Department of Pharmacy, Veterans Administration Medical Center, Ann Arbor, Department of Surgery, University of Michigan, Ann Arbor, Department of Pharmacy Administration, College of Pharmacy, Department of Health Services Management and Policy, School of Public Health, University of Michigan, Ann Arbor, Michigan
\end{abstract}

\begin{abstract}
Efficacy and safety of intravenous and intramuscular vitamin $K$ were compared prospectively in patients receiving total parenteral nutrition. Sixty patients randomly received either a 1-mg daily iv injection (iv group) or a 10-mg weekly injection group (im group). Efficacy was determined by the prolongation of twice-weekly prothrombin (PT) and activated partial thromboplastin (APTT) times. The prolongation of both was not significantly different between the im and iv groups. The percent of PTs outside the normal range was not different for the two groups, although the iv group had more APTT values outside the range than did the im group ( $p=$ 0.002 ). The number of adverse reactions reported in the iv (5)
\end{abstract}

and im (4) groups was also similar. Reactions were minor, not reproducible, and all patients recovered without sequelae. PT results from the iv and im groups were combined and compared to values from 28 patients in an earlier study who did not receive vitamin $K$. PTs in the no-vitamin $K$ group were significantly prolonged over the vitamin $\mathrm{K}$ group $(p=0.0004)$. The results confirm that regular addition of vitamin $K$ to TPN regimens decreases the incidence of elevated PTs. When administered appropriately, iv and im administration of vitamin $\mathrm{K}$ appear to be equally safe and effective in maintaining normal PT's and AP'TTs. (Journal of Parenteral and Enteral Nutrition 12:174-177, 1988)
Total parenteral nutrition (TPN) has been shown to be a safe and effective method of providing nutrition for patients unable to tolerate an adequate oral diet. ${ }^{1-3}$ TPN solutions commonly contain glucose, amino-acid mixtures, electrolytes, fat emulsions, trace elements, and vitamins including vitamin $\mathrm{K}^{4}$ Vitamin $\mathrm{K}$ is indicated for patients with coagulation disorders, malabsorption syndromes, and for those who are unable to maintain an adequate oral intake. ${ }^{5}$ Vitamin $\mathrm{K}$ is necessary for the liver synthesis of clotting factors II, VII, IX, and X which are essential for maintaining normal hemostasis. Minimum adult daily requirements range from $0.03-1.5 \mu \mathrm{g} /$ kg body weight. ${ }^{6}$

The association of vitamin $\mathrm{K}$ deficiency with bleeding abnormalities has been well documented. ${ }^{7-10}$ The oral preparation requires the presence of bile salts in the intestine for proper absorption. Because absorption is often unpredictable, the intramuscular or subcutaneous preparation has generally been preferred. Alternatively, vitamin $\mathrm{K}$ can be administered intravenously to improve prothrombin response rapidly in severe hemorrhagic states associated with hypoprothrombinemia, and to patients who fail to respond to other routes of administration. ${ }^{11-13}$

A previous study at our institution showed that patients who received TPN solutions for varying lengths of time and did not receive vitamin $\mathrm{K}$ demonstrated a high incidence of prolonged prothrombin times. ${ }^{14}$ Review of the literature for vitamin $\mathrm{K}$ requirements and admin-

\footnotetext{
Received for publication, November 10, 1986.

Accepted for publication, March 25, 1987.

Reprint requests: Gregory P. Schepers, Pharm. D., Veterans Administration Medical Center, 2215 Fuller Road, Ann Arbor, MI 48105.
}

istration guidelines revealed conflicting recommendations. Merck Sharp and Dohme, which manufacturers an injectable form of vitamin $K$, cautions that deaths have occurred after rapid intravenous administration and further states that transient flushing, taste disturbances, dizziness, rapid and weak pulses, profuse sweating, hypotension, dyspnea, and cyanosis have been reported after vitamin $\mathrm{K}$ injection. ${ }^{5}$ Whether these reactions were due to vitamin $\mathrm{K}$ or the vehicles used in commercial preparations is unclear. Their cautionary statements have been justified by other recent clinical evidence of adverse reactions following rapid intravenous injection of vitamin $\mathrm{K}$. There have been four case reports of patients receiving vitamin $\mathrm{K} 5$ - to $10-\mathrm{mg}$ iv over short periods of time (three patients, $2-10 \mathrm{~min}$, one patient, $45 \mathrm{~min}$ ) who subsequently experienced major adverse reactions. There were two episodes of cardiovascular collapse, one of severe hypotension, and one of generalized body tremors. ${ }^{15-18} \mathrm{Six}(0.5 \%)$ adverse reactions were documented in the Boston Collaborative Drug Surveillance Program (1966-1975) in which 1238 patients received vitamin $\mathrm{K}$. Two patients developed erythematous rashes, two injection-site complications, one a fever, and one a grand mal seizure. ${ }^{19}$

In spite of these cautions, vitamin $\mathrm{K}$ can safely be given intravenously particularly when it is not administered rapidly. Pridmore et al $^{20}$ described the uncomplicated regular intravenous administration of $5 \mathrm{mg}$ of vitamin $\mathrm{K}$ to 15 patients during pregnancy. Frick et $\mathrm{al}^{6}$ noted the safe use of small amounts of vitamin $K$ in 10 patients on starvation diets receiving antibiotic therapy. In addition, hospitals are beginning to add vitamin $\mathrm{K}$ to TPN solutions intended for intravenous administration. In light of these reports, questions remain concerning 
the safety, the optimal route of administration, and the daily requirement of vitamin $\mathrm{K}$. The specific aims of this study were to: (1). determine if a 1-mg intravenous dose of vitamin $\mathrm{K}$ daily is as effective as a weekly intramuscular $10-\mathrm{mg}$ dose in maintaining normal prothrombin and activated partial thromboplastin times. (2). determine if there is a difference in the incidence of adverse reactions among TPN patients receiving intravenous or intramuscular vitamin $K$. (3). confirm that regular addition of vitamin $\mathrm{K}$ to TPN regimens results in a lower incidence of abnormal prothrombin times.

\section{METHODS}

\section{Patient Selection}

From April 1985 to December 1985, 60 patients receiving total parenteral nutrition entered our prospective study. Patients received either $1 \mathrm{mg}$ of vitamin $\mathrm{K}$ per day in their TPN solutions (iv group) or a $10-\mathrm{mg}$ weekly intramuscular injection of vitamin $\mathrm{K}$ (im group). The vitamin $\mathrm{K}$ used in this study (Aquamephyton) was obtained from Merck Sharp and Dohme. Exclusion criteria were known hypersensitivity to vitamin $K$, history of a bleeding disorder, concurrent oral diet, administration of warfarin, vitamin $\mathrm{K}$ (other than protocol), or blood products 1 week prior to or during TPN; and receipt of TPN for less than 5 days.

In 1981, 28 patients at our institution who received TPN but did not receive vitamin $\mathrm{K}$ were studied by the TPN team. ${ }^{14}$ Data from the 28 patients were compared with that from the 60 patients (iv + im groups) in the present study to evaluate the effect of regular vitamin $K$ administration.

\section{Study Design}

Sixty patients were randomized into the intravenous or intramuscular group using the last digit of their social security number. Thirty-four patients received intravenous vitamin $\mathrm{K}$ and 26 received intramuscular. Prior to initiation of TPN therapy, the TPN team pharmacist reviewed the patients medical record to identify any medications or diseases that might influence vitamin $\mathrm{K}$ response. The primary physician was notified concerning the proposed route of administration or ineligibility for the study. The study was carefully explained to patients and written consent was obtained. Patients who were ineligible or declined to participate received the standard institutional TPN solutions.

To determine the effectiveness of the vitamin $\mathrm{K}$ administration in the iv and im groups, prothrombin times (PT) and activated partial thromboplastin times (APTT) were taken twice a week. The mean PT reference value at our institution is $10.7 \mathrm{sec}$ (range $8.4-13 \mathrm{sec}$ ). Our mean APTT reference value is $26.4 \mathrm{sec}$ (range $22-31 \mathrm{sec}$ ). The difference of the PT and APTT times above the reference mean values was used to calculate PT or APTT prolongation of each group. In addition, serum bilirubin, alkaline phosphatase, and serum liver transaminases were drawn at least weekly to assess liver function. The incidence of vitamin $\mathrm{K}$ adverse reactions following iv or im administration was determined by daily patient inter- views and medical record review. In this study an adverse reaction was defined as an unexplained report that had been previously attributed by the manufacturer to vitamin $\mathrm{K}$ iv or im administration. Study data was collected beginning on the date of the first inclusion of vitamin $\mathrm{K}$ in the TPN solutions (iv group) or on the date of the first intramuscular injection (im group) and was continued at daily intervals until TPN was discontinued.

\section{Statistical Analysis}

Differences between groups were analyzed using twotailed $t$-tests (age, pretreatment percent ideal body weight, duration of parenteral nutrition, PT and APTT prolongation), chi-squared tests (days treated by medical or surgical services, days of peripheral or central TPN, PT and APTT outside the normal range), and Fisher's exact tests (sex, race, vitamin $\mathrm{K}$ side effects).

\section{RESULTS}

The safety of vitamin $\mathrm{K}$ administration was compared in the iv and im groups. Efficacy was also determined in the two groups using PT and APTT times. Efficacy data from the two groups in the present study was combined and compared to data from the 1981 study (no vitamin $\mathrm{K})$.

\section{Treatment Groups}

Characteristics of the iv and im groups as well as similar data from the previous study are shown in Table I. The iv and im groups were similar in age, sex, pretreat-

TABLE I

Characteristics of the 88 patients entered into vitamin $K$ study

\begin{tabular}{|c|c|c|c|}
\hline & iv group & im group & $\begin{array}{l}\text { No vitamin } K \\
1981 \text { study }\end{array}$ \\
\hline Age (year) & $60 \pm 12$ & $63 \pm 9$ & $60 \pm 15.6$ \\
\hline \multicolumn{4}{|l|}{ Sex } \\
\hline Males & 33 & 26 & 27 \\
\hline Females & 1 & $\mathbf{0}$ & 1 \\
\hline \multicolumn{4}{|l|}{ Race } \\
\hline Black & 4 & 2 & 3 \\
\hline Caucasian & 30 & 24 & 25 \\
\hline Pretreatment \% IBW & $101 \pm 25$ & $107 \pm 21$ & $90 \pm 18$ \\
\hline $\begin{array}{l}\text { \% Days medicine serv- } \\
\text { ice }^{\alpha}\end{array}$ & 34.3 & 16.7 & 35.6 \\
\hline$\%$ Days surgical service ${ }^{a}$ & 65.7 & 83.3 & 64.4 \\
\hline TPN duration (days) & $13.3 \pm 8.44$ & $13.3 \pm 7.37$ & $12.4 \pm 9.2$ \\
\hline$\%$ Days central TPN ${ }^{b}$ & 74.9 & 81.2 & 63.2 \\
\hline $\begin{array}{l}\text { \% Days peripheral } \\
\text { TPN }^{b}\end{array}$ & 25.1 & 18.8 & 36.8 \\
\hline $\begin{array}{l}\text { Patients on oral anti- } \\
\text { biotics }\end{array}$ & 1 & 0 & \\
\hline $\begin{array}{l}\text { Low-dose heparin pa- } \\
\text { tients }\end{array}$ & 10 & 7 & \\
\hline Liver disease patients & 4 & 3 & \\
\hline Deaths during TPN & 1 & 0 & 2 \\
\hline
\end{tabular}

$x^{2}, t$-test, Fisher's exact test, no significant difference except:

${ }^{a} \%$ days medical $v s$ surgical service:

$$
\begin{aligned}
& \text { iv }>\text { im } \chi^{2}=30.23, p<0.0001 \\
& \text { No-vit } K>\text { iv }+ \text { im } \chi^{2}=8.90, p=0.003
\end{aligned}
$$

${ }^{b} \%$ days central $u s$ peripheral TPN

$$
\text { im }>\text { iv } \chi^{2}=4.00, p=0.05
$$

iv + im $>$ No-vit $K \chi^{2}=24.97, p=0.0001$. 
ment percent ideal body weight, and duration of TPN. The percentage of surgical service patient days was higher in the im group than in the iv group $(p<0.0001)$. Percent days on central ( $v s$ peripheral) TPN was also higher in the im group $(p=0.05)$. Because the ordering of PT and APTT times was controlled by the investigators, there should not be a service effect. In addition, the efficacy and safety of intravenous vitamin $\mathrm{K}$ administration has not been observed to be influenced by central or peripheral routes.

The groups had a similar distribution of underlying diseases (Table II). During the 8-month study, 24 of a potential 84 patients who received TPN were excluded. The reasons for exclusions are listed in Table III. Only two patients declined to participate in the study; other exclusions were for not meeting study criteria.

To evaluate differences in TPN populations which received or did not receive vitamin $K$, the iv and im groups were combined then compared to the 1981 novitamin $\mathrm{K}$ group. No differences in age, sex, pretreatment percent ideal body weight, and duration of TPN were seen. The percentage of surgical TPN days $(p=0.003)$ and days on central TPN ( $p=0.0001)$ were higher in the iv + im group than in the no-vitamin $K$ group.

\section{Effectiveness of Vitamin K Administration}

PT and APTT times from the iv and im groups were compared to determine the effectiveness of the two routes of administration (Table IV). The mean prothrombin time prolongation of the iv and im group were not significantly different. Similarly the percentage of PT times outside the normal range was not statistically different. Prolongation of APTT times was similar in the iv and

TABLE II

Underlying diseases of the 88 patients entered into the vitamin $K$ study

\begin{tabular}{lrcc}
\hline & iv group & im group & $\begin{array}{c}\text { No vitamin K } \\
\text { 1981 study }\end{array}$ \\
\hline Cancer & 16 & 9 & 10 \\
Head/neck/lung & 5 & 2 & 4 \\
Gastrointestinal & 10 & 5 & 5 \\
Bladder & 1 & 2 & 1 \\
GI obstruction & 9 & 10 & 10 \\
Pancreatitis & 2 & 1 & 2 \\
Intraabdominal abscess & 2 & 2 & 1 \\
Abdominal aortic aneu- & 1 & 1 & 1 \\
$\quad$ rysm & 1 & 1 & \\
COPD & 1 & 1 & 2 \\
Crohns/ulcerative colitis & 1 & 1 & 1 \\
Gastrointestinal bleeding & 1 & 0 & 1 \\
Malnourishment & 34 & 26 & 28 \\
Total patients & & &
\end{tabular}

TABLE III

Reasons for vitamin $K$ study patient exclusions

\begin{tabular}{lr}
\hline \multicolumn{1}{c}{ Reason } & No. \\
\hline Blood products administration & 8 \\
Concurrent oral diet & 3 \\
TPN less than 5 days & 7 \\
Vitamin K (off-protocol) & 2 \\
Warfarin administration & 2 \\
Patient declined & 2 \\
Total & 24 \\
\hline
\end{tabular}

im group, although, the iv group had a significantly higher percentage of APTT times outside the normal range $(p=0.002)$.

In this study 17 patients ( $10 \mathrm{iv}$, seven im) received low-dose subcutaneous heparin with TPN. To analyze the potential impact of heparin on blood clotting tests, the APTT time prolongation of the heparin group was compared to that of the nonheparin group. No statistical difference was seen.

To confirm that regular administration of vitamin $\mathrm{K}$ to patients on TPN was effective in maintaining normal PT times, the combined iv + im group was compared to the no-vitamin $\mathrm{K}$ group. The mean prolongation of the no-vitamin $\mathrm{K}$ group $(3.7 \mathrm{sec})$ was significantly greater than that of the vitamin K group $(1.9 \mathrm{sec})(p=0.0004)$. Similarly, the no-vitamin $\mathrm{K}$ group had a higher percentage of PT times outside the normal range $(p=0.002)$. Because APTT times were not recorded in the previous study, comparison between patients in the vitamin $\mathrm{K}$ group and no-vitamin $\mathrm{K}$ group was not possible.

\section{Adverse Reactions}

The number of adverse reactions reported in the iv and im groups was similar (Table V). Five side effects (14.7\% of patients) were reported in the iv group and four $(\mathbf{1 5 . 4 \% )}$ in the im group. All reactions were minor and the patients recovered without sequelae. No study patient had more than one adverse reaction. The iv group received 345 solutions containing vitamin $\mathrm{K}$. Forty-eight intramuscular injections were administered to the im group.

TABLE IV

Effect of vitamin $K$ on PT and APTT times

\begin{tabular}{|c|c|c|c|}
\hline & iv group & im group & $\begin{array}{l}\text { No vitamin } K \\
1981 \text { study }\end{array}$ \\
\hline $\begin{array}{l}\text { Mean PT prolongation } \\
(\mathrm{sec}) \text { (above } 10.7 \mathrm{sec})\end{array}$ & $1.4 \pm 1.3$ & $1.7 \pm 1.8$ & $3.7 \pm 4.3^{a}$ \\
\hline No. $(\%)$ of PTs $>13(\mathrm{sec})$ & $27(22.3)$ & $26(29.2)$ & $33(42.9)^{b}$ \\
\hline Total no. of PTs & 121 & 89 & 77 \\
\hline PT frequency (days) & 3.8 & 3.9 & 4.5 \\
\hline $\begin{array}{l}\text { Mean APTT prolongation } \\
\text { (sec) (above } 26.4 \mathrm{sec} \text { ) }\end{array}$ & $4.3 \pm 4.9$ & $3.5 \pm 9.2$ & \\
\hline $\begin{array}{l}\text { No. (\%) of APTTs > } 31 \\
\quad(\mathrm{sec})\end{array}$ & $44(36.4)$ & $14(15.7)^{c}$ & \\
\hline Total no. of APTTs & 121 & 89 & \\
\hline APTT frequency (days) & 3.75 & 3.90 & \\
\hline Patients & 34 & 26 & 28 \\
\hline \multicolumn{4}{|c|}{$\begin{array}{l}\chi^{2}, t \text {-test, no significant difference except: } \\
{ }_{a} \text { PT prolongation: No-vitamin } \mathrm{K}>\mathrm{iv}+\mathrm{im} p=0.0004 \\
{ }^{b} \text { PT }>13 \text { sec: no-vitamin } \mathrm{K}>\mathrm{iv}+\mathrm{im} \chi^{2}=9.435, p=0.002 \\
{ }^{\mathrm{C}} \text { APTT }>31 \text { sec: } \mathrm{iv}>\mathrm{im} \chi^{2}=9.68, p=0.002\end{array}$} \\
\hline \multicolumn{4}{|c|}{$\begin{array}{c}\text { TABLE V } \\
\text { Vitamin K side effects }\end{array}$} \\
\hline & \multicolumn{2}{|c|}{ iv group } & im group \\
\hline Diaphoresis & & & 1 \\
\hline Dizziness & 1 & & 2 \\
\hline GI bleed & 1 & & 1 \\
\hline Hematoma & 1 & & \\
\hline Melena & 1 & & \\
\hline Chest pain (mild) & 1 & & \\
\hline Total & $5(14$ & & $(15.4 \%)^{a}$ \\
\hline
\end{tabular}

${ }^{a}$ Percent of patients having side effects. 


\section{DISCUSSION}

\section{Effect of $i v$ and im Vitamin $K$}

Since the liver constantly produces coagulation factors, daily rather than weekly administration of vitamin $\mathrm{K}$ would seem preferrable. This study provides evidence that iv and im administration of vitamin $\mathrm{K}$ given appropriately are equally effective in maintaining normal PT and APT'T times. The depot effect of the im injection, the storage of vitamin $\mathrm{K}$ in the liver, the long half-life of the clotting factors, and the small daily requirement of vitamin $\mathrm{K}$ may all contribute to the apparent equalizing effect seen in our study. In spite of the pharmacokinetic differences, our regimens were found to be equally effective.

Administration guidelines for vitamin $\mathrm{K}$ should be determined by each hospital's nutrition team. Infusion with daily TPN solutions would eliminate the additional discomfort of an intramuscular injection. As demonstrated, routine administration of vitamin $\mathrm{K}$ and blood coagulation monitoring should decrease the frequency of elevated PT and APTT times.

Administration of low doses of heparin did not affect APTT times in either the iv or im group. This supports earlier observations that mini-dose heparin has a minimal effect on APTT times. ${ }^{21}$

\section{Effect of Vitamin K on Prothrombin Time}

Regular addition of vitamin $\mathrm{K}$ to TPN regimens has been shown in this study to decrease the incidence of hypoprothrombinemia. A lower frequency of elevated PT times was seen in the combined iv + im group when compared to the no-vitamin $\mathrm{K}$ group. Whether the increased incidence of normal clotting times is clinically significant has not been demonstrated in this study. However the association between elevated PT times and the risk of hemorrhagic abnormalities (eg, bleeding, bruising) has been well documented..$^{5-7}$ Rational therapy would seem to include regular vitamin $\mathrm{K}$ administration to patients who are on prolonged TPN therapy.

\section{Adverse Reactions}

The frequency of adverse reactions in the iv and im groups was not statistically different. Three of the five adverse reactions reported in the iv group (GI bleed, hematoma, melana) were of more serious nature compared to one of four in the im group (GI bleed). The reactions encountered were minor, short in duration, and patients recovered without sequelae. Adverse reactions were not reproducible with continued vitamin $\mathrm{K}$ administration. In this study $1 \mathrm{mg}$ of iv vitamin $\mathrm{K}$ was infused over at least 8-12 hr. In contrast to clinical reports mentioned earlier which involved more rapid administration, episodes of cardiovascular collapse and hypersensitivity reactions were not seen.

The association of serious adverse reactions with intravenous or intramuscular vitamin $\mathrm{K}$ is not well defined.
Case reports provide useful clinical information but cannot describe an incidence. One serious adverse reaction (grand mal seizure) was reported in the Boston Collaborative Drug Surveillance of 1238 patients $(0.08 \%$ incidence). Although no serious adverse reactions were seen in the 60 study patients, this possibility cannot be ruled out since the incidence is very low.

The results confirm previous findings that vitamin $\mathrm{K}$ is of value in maintaining normal blood clotting times in TPN patients. Our study also provides evidence to support the clinical reports that the intravenous route is a safe and effective way to administer low doses of vitamin $\mathrm{K}$ over extended periods of time.

\section{REFERENCES}

1. Dudrick SJ, Wilmore DW, Vars HM, et al: Long-term total parenteral nutrition with growth development, and positive nitrogen balance. Surgery 64:134-42, 1968

2. Wilmore DW, Dudrick SJ, Vars HM, et al: Long-term intravenous hyperalimentation. Fed Proc 27:486, 1968

3. Filler RM, Eraklis AJ, Rubin VG, et al: Long-term intravenous hyperalimentation in infants. N Engl J Med 281:589-594, 1969

4. American Medical Association: Guidelines for essential trace element preparations for parenteral use. JPEN 3:263-267, 1979

5. Aquamephyton package insert. Merck Sharp and Dohme, West Point, PA; 1982

6. Frick PG, Riedler G, Brogli $\mathrm{H}$ : Dose response and minimal daily requirement of vitamin $K$ in man. J Appl Physiol 23:387-389, 1967

7. Vitamin $\mathrm{K}$ deficiency in adults. Nutr Rev 26:165-167, 1968

8. Blanchard RA, Furie BC, Jorgensen M, et al: Acquired vitamin $K$ dependent carboxylation deficiency in liver disease. Engl $\mathrm{J}$ Med 305:242-248, 1981

9. Kelton JG, Hirsh J: Bleeding associated with antithrombic therapy. Semin Hematol 17:259, 1980

10. Gilman AG, Goodman LS, Gilman A, eds: The Pharmacological Basis of Therapeutics, 6th ed. MacMillan, New York, 1980, pp 1592-1593

11. Butler VE, O'Donnell J: Vitamin K preparations: Guidelines and cautions for intravenous use. Infusion 5:154-155, 1981

12. Trissel LA: Phytonadione. IN Handbook of Injectable Drugs, 3rd ed. American Society of Hospital Pharmacists Inc. Bethesda, MD, 1983 , pp 384-387

13. American Society of Hospital Pharmacist: Drug informationPhytonadione. American Society of Hospital Pharmacist, Bethesda, MD, 1986, pp 1871-1873

14. Dalton MJ, Schepers GP, Gee JP, et al: Consultative total parenteral nutrition teams: The effect on the incidence of total parenteral nutrition-related complications. JPEN 8:146-152, 1984

15. Mattea FJ, Quinn K: Adverse reactions after intravenous phytonadione administration. Hosp Pharm 16:224-235, 1981

16. Bjornsson TD, Blaschke TF: Vitamin $\mathrm{K}$ disposition and therapy of warfarin overdose. Lancet $2: 846-847,1978$

17. Barash P, Kitahata LM, Mandel S: Acute cardiovascular collapse after intravenous phytonadione. Anesth Analg 55:304-306, 1976

18. Rich EC, Drage CW: Severe complications of intravenous phytonadione therapy. Postgrad Med 72:303-306, 1982

19. Miller RR, Greenblatt DJ: Drug effects in hospitalized patientsExperiences of the Boston Collaborative Drug Surveillance Program: 1966-1975. Wiley, New York, 1976, pp 108-109

20. Pridmore BR, Murray KH, McAllen PM: The management of anticoagulant therapy during and after pregnancy. $\mathrm{Br} J$ Obstet Gynaecol 82:740-744, 1975

21. Sherry S, Belber A: Anticoagulants, platelet-controlling drugs and thrombolytic agents. IN The Heart, 6th ed. McGraw-Hill Inc, New York, 1986, pp 1667-1669 